\title{
Landau Ghosts and Anti-Ghosts in Condensed Matter and High Density Hadronic Matter
}

\author{
S. Sivasubramanian, Y. N. Srivastava ${ }^{\dagger}$, A. Widom \\ Physics Department, Northeastern University, Boston MA USA \\ and \\ ${ }^{\dagger}$ Physics Department 83 INFN, University of Perugia, Perugia IT
}

October 29, 2018

\begin{abstract}
It is observed that the "ghost" (originally discovered by Landau in quantum electro-dynamics) and its counterparts in other theories are indeed ubiquitous as they occur in a one-loop approximation to any conventional (unbroken) gauge theory. The mechanism is first exposed in its generality via the Dyson equation and a simple but explicit example in condensed matter is provided through the static Clausius-Mossotti and its dynamic counterpart the Lorenz-Lorentz equation. The physical phase transition phenomenon associated with it is found to be super-radiance. We verify quantitatively that water (and many other polar liquids) are indeed super-radiant at room temperature. In quantum chromo-dynamics on the other hand, we encounter, thanks to asymptotic freedom, an "anti-ghost" which is closely associated with color confinement. Thus, in QCD, free quarks and glue exist in a super-radiant phase and hadronic matter in the normal phase.
\end{abstract}




\section{Introduction}

It was discovered many decades ago[1], that the dimensionless coupling constant in QED, $\alpha_{0}=e_{0}^{2} / \hbar c$, after 1-loop renormalization becomes momentum transfer $Q^{2}$ dependent $\alpha\left(Q^{2}\right)$ and that it has a pole at a (space-like $Q^{2}>0$ ) value which is clearly unphysical. A Landau ghost (or pole) is the name associated with such a singularity. If $\alpha\left(Q^{2}\right)$ can turn negative either by passing through a zero or through a pole, it would lead to instability separating a phase where two like charges repel to a different phase where they attract. In the present work, we shall explore a general origin of the ghost (and similar pathologies) via the Dyson equation. While for QED, the phase transition is at unattainable high energies, in certain condensed matter situations the singularity is at very low energies and leads to an observable phase transition called super-radiance. A very practical application is to the dielectric properties of systems for which a one loop renormalization leads to Clausius-Mossotti(CM) and Lorenz-Lorentz(LL) equations. For such phenomenological models, the phase transition computations can be done to completion [2, 3, 4, 5] and we find quantitatively that water is indeed superradiant at room temperature, a result predicted earlier by other authors from somewhat different theoretical considerations [6].

In contrast to QED with a Landau ghost, in the same 1-loop approximation, a non-abelian gauge theory such as QCD for quarks and glue avoids the Landau ghost (provided the number of quark flavors is limited to 16) and is asymptotically free (AF). However, this welcome change in sign of the beta function leading to AF brings with it its own pathology. The Landau pole is now no longer a pole on the real axis in the physical region $\left(s=-Q^{2}>0\right)$ since it acquires an imaginary part, but the sign of the imaginary part is wrong i.e., it is negative. It seems appropriate to call this latter phenomenon an "anti-ghost" since, as we shall see later it also signals an instability. Physically, it leads to the pleasing result [7 that quark and gluon states can only be transient realized under restricted conditions (of large energies and momentum transfers). We shall not consider here how color confinement with normal hadronic phase prevails at low energies and momentum transfers.

The paper is organized as follows. In Sec.2, the genesis of the singularity is considered through a generic discussion of "feedback" which is at the heart of the Dyson equation. In Sec.3, we first develop the CM and LL equations for dielectrics in order to exhibit the resulting phase transition as critical values (of the volume and/or the temperature) leading to super-radiance are reached for the important case of water (and other polar materials). 
In Sec.4, we briefly discuss QCD and what the anti ghost entails for the resulting physics there. Sec.5 closes the paper with some concluding remarks and directions for future work.

\section{Dyson equation and the critical feedback}

Let $D$ be the exact photon propagator, $D_{0}$ the free photon propagator and $\Pi$ its proper self energy part 8] (suppressing all Lorentz indices and momentum labels momentarily). The Dyson equation 90 then reads

$$
D=D_{0}+D_{0} \Pi D=\left(1-D_{0} \Pi\right)^{-1} D_{0},
$$

or, equivalently written in terms of the inverse propagators

$$
D^{-1}=D_{0}^{-1}-\Pi \text {. }
$$

For computations to follow, it would be useful to recast the logic behind Eq.(11) in the language of linear feedback. Suppose an external current $\delta J_{\text {ext }}$ is applied to a system and we wish to find the corresponding change in the potential from $A$ to $A+\delta A$. The potential change $\delta A$ is given in terms of the total change in the current $\delta J$ through the free propagator $D_{0}$ by

$$
\delta A=D_{0} \delta J=D_{0}\left(\delta J_{e x t}+\delta J_{i n d}\right),
$$

where in Eq.(3), the total change in the current contains a feedback current $\delta J_{i n d}$ in addition to the applied $\delta J_{\text {ext }}$. The induced piece is the system's linear response to the change in the potential through the "self energy" term $\Pi$

$$
\delta J_{\text {ind }}=\Pi \delta A .
$$

Now defining the exact propagator through

$$
\delta A=D \delta J_{\text {ext }},
$$

Eq.(11) follows from Eqs.(355). Eq.(1), when depicted pictorially as in Fig. [1], makes abundantly clear the linear feedback mechanism implicit in the Dyson equation.

Restoring explicitly the Lorentz indices, we may write the Feynman gauge, renormalized photon propagator of 4- momentum $q$ with

$$
s=q^{2}=(\omega / c)^{2}-|\mathbf{k}|^{2}
$$




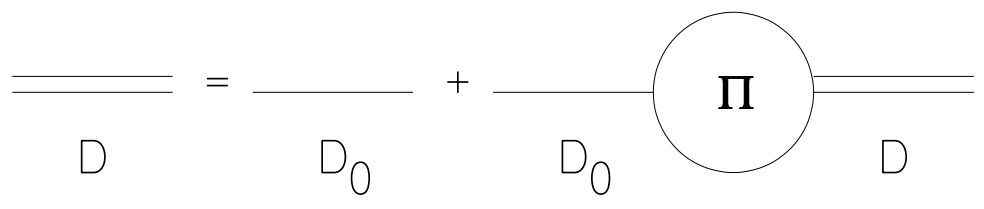

Figure 1: The Dyson equation for the exact propagator $D$ is shown as a linear feedback to the free propagator $D_{0}$ through the "self energy" term $\Pi$.

( $\omega$ the photon frequency and $\mathbf{k}$ its wave-vector), in terms of the "running coupling constant" $\alpha(s)$ and the dielectric "tensor" $\epsilon(s)$ as follows:

$$
\alpha_{0} D^{\mu \nu}(q)=\alpha(s) D_{0}^{\mu \nu}(q)=\frac{\alpha_{0} D_{0}^{\mu \nu}(q)}{\epsilon(s)} .
$$

Clearly, both poles and zeroes in $\alpha$ (which become respectively, the zeroes and poles of $\epsilon$ ) are pathological.

For a renormalization at an arbitrary space-like point $\left(s=-\Lambda^{2}\right)$,

$$
\Pi_{\mu \nu}(q)=\left[-4 \pi \alpha\left(-\Lambda^{2}\right)\right]\left(q_{\mu} q_{\nu}-\eta_{\mu \nu} q^{2}\right) \pi_{c}(s) .
$$

With $\pi_{c}\left(s=-\Lambda^{2}\right)=0$ we have

$$
\alpha(s)=\left[\frac{\alpha\left(-\Lambda^{2}\right)}{1+4 \pi \alpha\left(-\Lambda^{2}\right) \pi_{c}(s)}\right]
$$

and likewise

$$
\epsilon(s)=1+4 \pi \alpha\left(-\Lambda^{2}\right) \pi_{c}(s) .
$$

Now let us consider the allowed singularities in the exact propagator $D_{\mu \nu}(q)$. There are branch point singularities in $D$ (for time-like momenta at all production thresholds) through unitarity as $\pi_{c}(s)$ develops an imaginary part. In the physical region of time-like momenta, there may even be poles corresponding to resonances on the unphysical Riemann sheet(s). On the other hand, for space-like momenta, $D$ is forbidden to have any poles, i.e., on general grounds, we must require that

$$
4 \pi \alpha\left(-\Lambda^{2}\right) \pi_{c}(s)>-1, \text { all } s<0
$$


(all space-like momenta for which $\omega<c|\mathbf{k}|$ ). Whenever this inequality is not satisfied, we shall have an unphysical Landau pole. The feedback is then beyond its critical value invalidating linear response and it would signal a phase transition to another, super-radiant phase of the theory[3].

For example, in one fermion loop QED, renormalized on shell at $s=0$, the standard vacuum result reads

$$
\epsilon_{1-\text { loop }}(s)=1-\frac{2 \alpha}{\pi} \int_{0}^{\infty}\left(z-z^{2}\right) \ln \left[1-\frac{s z(1-z)}{\kappa^{2}-i 0^{+}}\right] d z
$$

where $\kappa=m c / \hbar$, with $m$ the fermion mass. In the space-like region $\epsilon$ decreases to zero at the Landau value

$$
-s_{L}=Q_{L}^{2}=\kappa^{2} e^{3 \pi / \alpha},
$$

beyond which it turns negative. Thus, QED is in its normal (stable) phase until an enormously large $Q^{2}$ value above which it would turn super-radiant. In the condensed matter example treated in the next section, a similar phenomenon would occur (this time through a zero in $\alpha$ ) but at physically relevant energies and hence of practical importance.

A discussion of the complementary notion of the anti-ghost is better done via a subtracted dispersion relation for $\epsilon$

$$
\epsilon(s)=1+\left(\frac{s+\Lambda^{2}}{\pi}\right) \int_{0}^{\infty} \frac{\left(d s^{\prime}\right) \Im m \epsilon\left(s^{\prime}\right)}{\left(s^{\prime}+\Lambda^{2}\right)\left(s^{\prime}-s-i 0^{+}\right)},
$$

In spinor QED, where only fermion loops contribute, $\Im m \epsilon(s)$ is positive definite ( for all physical $s$ ). In one loop, it is given by

$$
\Im m \epsilon(s)=\frac{\vartheta\left(s-4 \kappa^{2}\right) \alpha}{3}\left[1+\frac{2 \kappa^{2}}{s}\right] \sqrt{\left[1-\frac{4 \kappa^{2}}{s}\right]},
$$

thus the system is "dissipative". Whenever the system is dissipative, the subtraction tells us ( in 1-loop) that there is a Landau ghost in the space like region.

On the other hand, for QCD, along with the fermion loops (quarks in this case) as above, which are dissipative, we also have a larger imaginary part with a negative sign from the gluons

$$
\Im m \epsilon(s)=\Im m \epsilon_{\text {glue }}(s)+\Im m \epsilon_{\text {quark }}(s),
$$

where

$$
\Im m \epsilon_{\text {glue }}(s)=-\vartheta(s)\left(\frac{11 \alpha_{s}}{8}\right)
$$


and

$$
\Im m \epsilon_{\text {quark }}(s)=\sum_{i=1}^{N_{f}} \vartheta\left(s-4 \kappa_{i}^{2}\right)\left(\frac{\alpha_{s}}{12}\right)\left[1+\frac{2 \kappa_{i}^{2}}{s}\right] \sqrt{\left[1-\frac{4 \kappa_{i}^{2}}{s}\right]}
$$

For the number of flavors $N_{f}<16$, the total imaginary part remains negative definite. This cures the Landau ghost problem and as well known we have asymptotic freedom, for in the space-like region $s=-Q^{2}<-\Lambda^{2}$,

$$
\epsilon\left(-Q^{2}\right)=1+\frac{\left(Q^{2}-\Lambda^{2}\right)}{\pi} \int_{0}^{\infty} \frac{d s[-\Im m \epsilon(s)]}{\left(s+\Lambda^{2}\right)\left(s+Q^{2}\right)}>1 .
$$

The price paid for exorcizing the Landau ghost into a benevolent anti-ghost is to turn the system into being non-dissipative i.e., into an "amplifier" [0] which is well known to be transient. We shall return to this problem in Sec.4.

\section{Landau ghost in condensed matter and super- radiance}

As stated earlier, unlike the Landau ghost in QED, in condensed matter we have unphysical singularities at low frequencies which signal phase transitions of practical importance for the physics and the chemistry of substances.

In condensed matter literature, the analogue of $\alpha \pi_{c}$ in Eq.(8) is known as the electric susceptibility $\chi_{e}$ and the object here is to relate it to macroscopic quantities such as the polarizability of the molecules $\hat{\alpha}$ and $v$ the volume per molecule. ( We have defined the polarizability with a "hat" in order to distinguish it from the coupling constant $\alpha$ ).

The CM equation 10, 11] is an ideal example of a linear feedback paralleling the argument of the last section. To briefly recall what is involved and to set the notation, the electric dipole moment per unit volume or, polarization $\mathbf{P}$

$$
\mathbf{P}=\chi_{e} \mathbf{E}=N\left\langle\mathbf{p}_{m o l}\right\rangle,
$$

where $N$ is the number of molecules per unit volume and $\left\langle\mathbf{p}_{\text {mol }}\right\rangle$ is the mean dipole moment of the molecules. Then, without the "feedback", justifiable in rare media where molecular separations are large, one assumes that the macroscopic field $\mathbf{E}$ is the same as that acting on a given molecule

$$
\left\langle\mathbf{p}_{\text {mol }}\right\rangle=\hat{\alpha} \mathbf{E},
$$


in terms of the polarizability. So that for rare media

$$
\chi_{e}=N \hat{\alpha}=\hat{\alpha} / v,
$$

where $v$ is the volume occupied by a single molecule.

In denser media where molecular separations are small, the polarization $\mathbf{P}$ induces an internal electric field $\mathbf{E}_{i}$. If one includes only the contributions from molecules far away, this feedback term is estimated to be

$$
\mathbf{E}_{i}=\left(\frac{4 \pi}{3}\right) \mathbf{P}
$$

Now, Eq.(19) leads to

$$
\begin{aligned}
\mathbf{P} & =N\langle\mathbf{p}\rangle=N \hat{\alpha}\left(\mathbf{E}+\mathbf{E}_{i}\right) \\
& =N \hat{\alpha}\left(\mathbf{E}+\frac{4 \pi}{3} \mathbf{P}\right) \\
& =\left[\frac{N \hat{\alpha}}{1-(4 \pi / 3) N \hat{\alpha}}\right] \mathbf{E} .
\end{aligned}
$$

It is perhaps not surprising therefore, that the CM equation gives the same expression for $\chi_{e}$ as is obtained from the lowest order mean field theory 12, 13]

$$
\chi_{e}=\left(\frac{(\hat{\alpha} / v)}{1-(4 \pi \hat{\alpha} / 3 v)}\right)
$$

and for $\epsilon$ one finds

$$
\epsilon=\left(\frac{1+2(4 \pi \hat{\alpha} / 3 v)}{1-(4 \pi \hat{\alpha} / 3 v)}\right) .
$$

The normal phase would require for its stability that the static polarizability satisfy

$$
4 \pi \hat{\alpha}<3 v
$$

in order for no poles to develop in $\epsilon$ (or $\chi_{e}$ ). On the other hand, as the molecular density decreases below a critical value $v_{c}$, i.e., for

$$
v<v_{c}=\left(\frac{4 \pi}{3}\right) \hat{\alpha}
$$

the normal phase would become unstable yielding to a new stable superradiant phase of Dicke-Lieb- Preparata 14, 15, 16, 17, 18] models.

CM equations become the Lorenz-Lorentz (LL) equations [19, 20, 21, 22] as static $\hat{\alpha}, \chi_{e}$ and $\epsilon$ get generalized to a finite frequency $\omega$. Here one finds 
giant (macroscopic) Lamb shifts in frequency due to the "feedback". Let us suppose that a single atom with atomic number $Z$ has a strong resonance at a frequency $\omega_{\infty}$, so that

$$
\hat{\alpha}(\omega) \approx\left(\frac{Z e^{2}}{m}\right) \frac{1}{\omega_{\infty}^{2}-\omega^{2}-2 i \gamma \omega},
$$

where $m$ is the electron mass. Eqs (24) and (28) then imply

$$
\chi_{e}(\omega) \approx\left(\frac{Z e^{2}}{m}\right) \frac{1}{\omega_{0}^{2}-\omega^{2}-2 i \gamma \omega},
$$

where the "renormalized" resonant frequency $\omega_{0}$ is given by

$$
\omega_{0}^{2}=\omega_{\infty}^{2}-\left(\frac{4 \pi Z e^{2}}{3 m v}\right)=\omega_{\infty}^{2}\left[1-\left(\frac{4 \pi \hat{\alpha}}{3 v}\right)\right]
$$

where $\hat{\alpha} \equiv \hat{\alpha}(\omega=0)$

At the critical temperature $T_{c}$, the static polarizability stability condition given by Eq.(26) becomes

$$
\left(\frac{4 \pi \hat{\alpha}\left(T_{c}\right)}{3}\right)=v \quad \text { (critical point) }
$$

Dynamically, the renormalized frequency in Eq.(30) is driven to zero at the critical temperature

$$
\omega_{0}\left(T \rightarrow T_{c}+0^{+}\right)=0,
$$

which again leads to Eq.(31).

In previous work [⿶凵⿴囗十 , we have obtained the phase diagram through (2 and 4 level) model Hamiltonians which allow us to compute $\hat{\alpha}(T)$ for a single molecule. It is to be emphasized that perturbative corrections would shift the exact boundary of the super-radiant phase from its CM value but its occurrence is left intact.

Here we simply estimate the polarizability of water using the Debye model[23]

$$
\hat{\alpha}=\hat{\alpha}_{o}+\left(\frac{\mu^{2}}{3 k_{B} T}\right) \text {. }
$$

For water 24, 25]

$$
\hat{\alpha}_{0}=1.494 A^{3} \text { and } \mu=1.855 \times 10^{-18} \text { Gauss } \mathrm{cm}^{3} .
$$


This gives for the polarizability of water at room temperature

$$
\hat{\alpha}_{\text {water }} \approx 28.6962 \AA^{3} \text {. }
$$

From the molecular density of water, we find $v \approx 30.0137 \AA^{3}$, so that the super-radiance condition

$$
\frac{v_{c}}{v}=\left(\frac{4 \pi \hat{\alpha}}{3 v}\right) \approx 4.0054>1,
$$

is satisfied showing that water is indeed super-radiant at room temperature. Surely we expect numerical corrections to the above result. But the "margin of safety" is reasonable so that the claim about room temperature water being super-radiant is robust.

To show the efficacy of the above criterion, we have carried a similar analysis for a large number of polar and non-polar materials 26. The results shown in Fig. [2] confirm that at room temperatures, many polar liquids are super-radiant whereas non-polar materials are not.

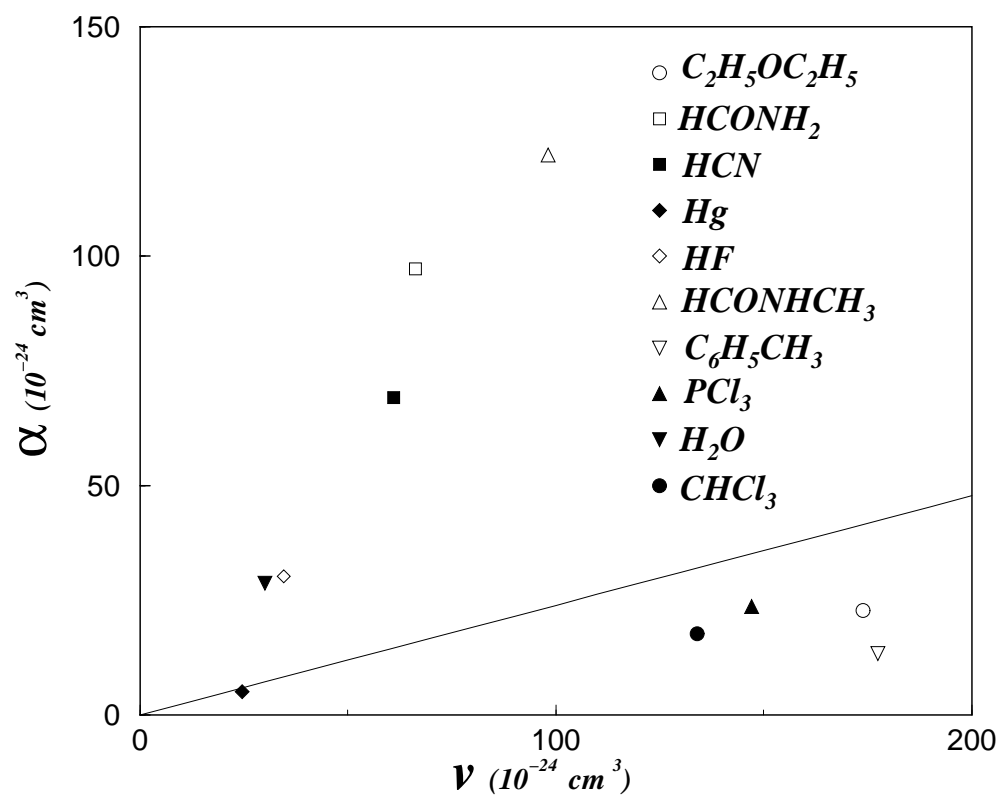

Figure 2: Polarizabilties $\hat{\alpha}$ for some materials are shown as a function of the volume per molecule $v$. Super-radiant materials lie above the line shown with slope $[3 /(4 \pi)]$. 


\section{Implications of the anti-ghost in QCD}

In this section, we resume our discussion of the physical implications arising from the negative sign of $\Im m \epsilon$ computed in 1-loop QCD. In general, such a negative sign signals a lack of causality, the latter driving the system to become an "amplifier" instead of a "dissipator". An amplifier (for example a laser with an inverted population entails the notion of a negative temperature) can only be a transient phenomenon since it requires a source to feed energy into it.

We may conclude from the above that the $J=1$, color octet channel of QCD represented by a gluon propagator with a negative absorptive part cannot be a part of the physical spectrum. We may conjecture that absorptive parts for all channels carrying color are of the amplifier variety thereby excluding all such states from the physical spectrum. At best then quarks and gluons can occur only transiently as virtual states in a restricted part of the phase space spanned by hadrons, the colorless children of quarks and glue. Only after one is able to produce hadrons as colorless composites of quarks

and gluons, would it be possible to check whether these true inhabitants of the "normal" phase of QCD have dissipative absorptive parts. Needless to say that we are nowhere near that goal.

\section{Conclusions and outlook}

We have presented a general discussion of the Landau ghost and related phenomena through the Dyson equation and these pathologies have been found to trigger phase transitions. A convincing case has been made for polar liquids to be in the super-radiant phase at ambient temperatures substantiating previous work on water. Similar arguments for QCD, through a wrong sign absorptive part in the gluon propagator, show that the gluons (and conjectured more generally for any channel carrying color) cannot be a part of the physical spectrum. This generalization needs verification. Also, further work is needed to show that the spectral functions for hadrons (that is the color singlet bound states) in QCD do possess positive absorptive parts as they must to qualify as the true "normal" ground states of the theory. 


\section{References}

[1] L.D. Landau, A.A. Abrikasov and I.M. Khalatnikov, Dokl. Akad. Nauk. USSR 95 497; 773; 1173 (1954); Nuovo Cimento Suppl. X 3, 80 (1956).

[2] S. Sivasubramanian, A. Widom and Y.N. Srivastava, Physica A301, 241 (2001).

[3] S. Sivasubramanian, A. Widom and Y.N. Srivastava, Int. J. Mod. Phy. B15, 537( 2001).

[4] S. Sivasubramanian, A. Widom and Y.N. Srivastava, arXiv: condmat/0209017.

[5] S. Sivasubramanian, A. Widom and Y.N. Srivastava, arXiv: condmat/0209617.

[6] E. Del Giudice, G. Preparata and G. Vitiello, Phys. Rev. Lett. 61, 1085 (1988).

[7] Y. Srivastava, S. Pacetti, G. Pancheri and A. Widom, in Proceedings of the " $e^{+} e^{-}$Physics at Intermediate Energies Workshop" at SLAC, Calif. eConf C010430, T19 (2001) .

[8] See, for example, J.D. Bjorken and S.D. Drell, "Relativistic Quantum Fields", McGraw Hill Book Company, NY (1965); chapter 19.

[9] F.J. Dyson, Phys Rev. 75, 1736(1949).

[10] O.F. Mossotti, Memorie Mat. Fis Modena 24, 49 (1850).

[11] R. Clausius, Die mechanische Wärmtheorie II 62 Braunschwieg (1897).

[12] S.E. Schanatterly and C. Tarrio, Rev. Mod. Phy.64, 619(1992)

[13] L. Fu and L. Resca, Phys. Rev. B47, 16194 (1993).

[14] K. Hepp and E.H. Lieb, Ann. Phys. 76, 360 (1973); Phys. Rev. A8, 2517 (1973).

[15] Y.K. Wang and F.T. Hoe, Phys. Rev. A7, 831 (1973).

[16] V.I. Emelanov and Yu.L. Klimontovich, Phys. Lett. A59, (1976).

[17] G. Preparata, "Quantum Coherence in Matter", World Scientific, Singapore (1990). 
[18] E. Del Giudice and G. Preparata, "A New QED Picture of Water", in "Macroscopic Quantum Coherence" Edited by E. Sassaroli, Y. Srivastava, J. Swain and A. Widom, World Scientific, Singapore (1998).

[19] V.L. Lorenz, Annalen Phys. 11, 70 (1880).

[20] H.A. Lorentz, Annalen Phys. 9, 641 (1880).

[21] C.M. Soukoulis, S. Datta and E.N. Economou, Phys. Rev. B49, 3800 ( 1994).

[22] B. Ersfeld and B.U. Felderhof, Phys. Rev. E57, 1118 (1998).

[23] P. Debye, "Polar Molecules", Dover Publications Inc., New York (USA) 1929.

[24] A. J. Russell and M. A. Spackman, Mol. Phys. 84, 1239 (1995).

[25] S. L. Shostak, W. L. Ebenstein and J. S. Muenter, J. Chem. Phys.94, $5875(1991)$.

[26] CRC handbook of chemistry and physics, CRC Press, Cleveland Ohio. (2001) 